\title{
Suppression of tissue harmonics for pulse-inversion contrast imaging using time reversal
}

\author{
Olivier Couture, Jean-François Aubry, Gabriel Montaldo, \\ Mickael Tanter and Mathias Fink
}

Laboratoire Ondes et Acoustique, ESPCI, 10 rue Vauquelin, 75005 Paris, France

E-mail: olicou@gmail.com

Received 29 April 2008, in final form 1 August 2008

Published 2 September 2008

Online at stacks.iop.org/PMB/53/5469

\begin{abstract}
Pulse-inversion (PI) sequences are sensitive to the nonlinear echoes from microbubbles allowing an improvement in the blood-to-tissue contrast. However, at larger mechanical indices, this contrast is reduced by harmonics produced during nonlinear propagation. A method for tissue harmonics cancellation exploiting time reversal is experimentally implemented using a 128-channel 12-bit emitter receiver. The probe calibration is performed by acquiring the nonlinear echo of a wire in water. These distorted pulses are timereversed, optimized and used for the PI imaging of a tissue phantom. Compared to normal (straight) pulses, the time-reversed distorted pulses reduced the tissue signal in PI by $11 \mathrm{~dB}$. The second harmonic signals from microbubbles flowing in a wall-less vessel were unaffected by the correction. This technique can thus increase the blood-to-tissue contrast ratio while keeping the pressure and the number of pulses constant.
\end{abstract}

(Some figures in this article are in colour only in the electronic version)

\section{Introduction}

The detection and quantitation of blood perfusion in an organ at the capillary level were made possible with the recent development of contrast agents and specialized pulse sequences (Burns and Wilson 2006). For example, these techniques are now used to assess the extent of hypoxic regions in the myocardium (Miller and Nanda 2004), the presence of angiogenic tumours in the liver (Albrecht et al 2003) and the modification of the perfusion of tumours after therapy (Cosgrove 2003).

In most cases, ultrasound contrast agents are microbubbles that are confined to the blood pool after injection. Their detection is done by exploiting their acoustic behaviour, which differs vastly from surrounding blood and tissue. For instance, these agents are resonant at specific ultrasound frequencies, they scatter ultrasound nonlinearly and they can be disrupted (de Jong et al 2000). In the past, several pulse sequences have been devised to separate 
microbubbles from tissue. Pulse inversion (PI), for example, uses two pulses of opposite phase (Simpson et al 1999, 2001). The sum of their respective echoes is non-zero only when nonlinear phenomena, such as scattering by microbubbles, are present. Combined with the disruption of the agents, PI allows the detection of slowly moving microbubbles replenishing tissue through capillaries (Wei et al 1998).

In addition to microbubble scattering, nonlinear echoes can also be produced during the propagation of high-pressure ultrasound pulses through a medium. Taking advantage of such nonlinear propagation, PI techniques have been used to improve contrast and resolution in ultrasound applications (Lencioni et al 2002, Tranquart et al 1999). However, nonlinear propagation is somewhat detrimental to the detection of microbubbles in deeper structures at high mechanical index (MI) since nonlinear echoes are no longer specific to the contrast agents.

Several solutions to improve contrast between microbubble harmonics and tissue harmonics have been proposed. New microbubble design has reduced the threshold of nonlinear scattering down to a pressure level which generates limited tissue harmonics. For example, compression-only microbubbles have an inherently asymmetrical oscillation even at low MI (de Jong et al 2007). Tissue harmonics was also reduced by imaging with higher harmonics (Bouakaz et al 2002) or by using amplitude modulation (Philipps and Gardner 2004).

Another possible solution to improve the contrast-to-tissue ratio (CTR) is tissue harmonics cancellation as proposed by Krishnan et al (1998). Their technique, based on active noise suppression, involves emitting a cancellation signal at twice the fundamental frequency of the main pulse. This cancellation signal, whose magnitude and phase must be carefully selected, interferes negatively with the second harmonic generated by the propagation of the wave through tissue. At a specific depth and pressure, this technique can cancel a large part of the tissue harmonics (theoretically, up to $30 \mathrm{~dB}$ ). This theoretical method was recently revisited by Krishnan and Thomenius (2008) and Pasovic et al (2008), numerically exploiting new developments in broader bandwidth transducers. However, this technique has yet to be applied experimentally.

Tissue harmonic cancellation can be seen as a consequence of the time invariance of the wave equation in the nonlinear regime, described by Tanter et al (2001). During nonlinear propagation, a purely sinusoid signal will be distorted, as the crests of the wave are moving faster than the troughs. Recording this signal at a given location, time-reversing it and sending it back in the opposite direction should theoretically result in the progressive recovery of the initial sinusoidal shape. Hence, using time-reversed distorted pulses for imaging should allow the cancellation of tissue harmonics at a specific depth and highlight the presence of microbubbles.

In this study, we describe the use of time reversal to cancel tissue harmonics in PI imaging at high MI. For this purpose, a fully programmable 12-bit transmitter receiver has been developed. Following a theoretical development, the acquisition of the distorted signal on a copper wire is depicted. Then minimization of tissue harmonics is described and the resulting images of flowing microbubbles in a wall-less tissue phantom are shown.

\section{Theory}

In a homogeneous medium, neglecting dissipative effects, acoustic wave propagation in a weakly nonlinear regime can be described by (Aanonsen and Barkve 1984)

$$
\nabla^{2} \phi-\frac{1}{c_{0}^{2}} \frac{\partial \phi}{\partial t^{2}}=\frac{1}{c_{0}^{2}} \frac{\partial}{\partial t}\left[(\nabla \phi)^{2}+\frac{1}{c_{0}^{2}} \frac{B}{A}\left(\frac{\partial \phi}{\partial t}\right)^{2}\right]
$$




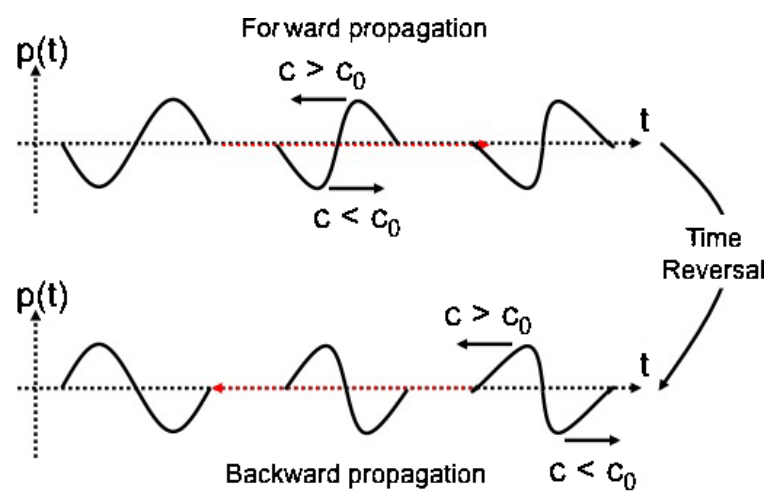

Figure 1. Time-reversal invariance in the nonlinear regime. Underpressures travel slower than overpressures. Time reversal inverts this lag, which is eliminated during back-propagation.

where $\phi(\vec{r}, t)$ is the velocity potential, which satisfies $\vec{v}=\nabla \phi, c_{0}$ is the speed of sound and $B / A$ is the nonlinearity parameter (Cobbold 2007). Here, the physical variables describing the propagation of an acoustic wave are the pressure and velocity fields, $p(\vec{r}, t)$ and $\vec{v}(\vec{r}, t)$, respectively.

Applying time reversal to a wave is equivalent to the following transformation:

$$
\left\{\begin{array}{l}
p(\vec{r}, t) \rightarrow p(\vec{r},-t) \\
\vec{v}(\vec{r}, t) \rightarrow-\vec{v}(\vec{r},-t)
\end{array} \quad \Leftrightarrow \quad \phi(\vec{r}, t) \rightarrow-\phi(\vec{r},-t) .\right.
$$

Mathematically, if $\phi(\vec{r}, t)$ is a solution of equation (1), then $\phi(\vec{r},-t)$ is also a solution of equation (1). This behaviour demonstrates the time-reversal invariance of the acoustic propagation in a nonlinear regime for propagation lengths smaller than the shock distance. Further theoretical considerations can be found in Tanter et al (2001).

Physically, this invariance means that harmonics generated by the propagation of an initially monochromatic field will progressively disappear after the time-reversal operation. Indeed, under nonlinear conditions, overpressures travel faster than underpressures. As shown in figure 1, due to time reversal, the troughs overtake their delay, which is then cancelled during back-propagation. This cancellation is complete in homogeneous and non-dissipative media. In viscous heterogeneous media, some of the original signal information is lost during propagation and therefore only part of the distortion can be cancelled through time reversal. However, this may still be enough to cancel relevant tissue harmonics.

\section{Method}

The time-reversal invariance can be applied to any type of pulse sequences affected by nonlinear propagation. For PI, our technique involves acquiring pulses of opposite phases distorted by nonlinear propagation, correcting them for frequency-dependent phenomena and imaging with these pulses after time reversal (TR) (figure 2). In this study, pulses that are affected by nonlinear propagation in the time domain are referred to as distorted pulses. Corrected pulses are the result of the time-reversal process, while straight pulses are monochromatic Gaussian pulses commonly used for imaging.

For the emission of these distorted pulses, each channel of the array had to be equipped with a high dynamic-range arbitrary waveform generator. A newly developed fully programmable 128-channel pulser receiver was developed in collaboration with LeCoeur Electronique 


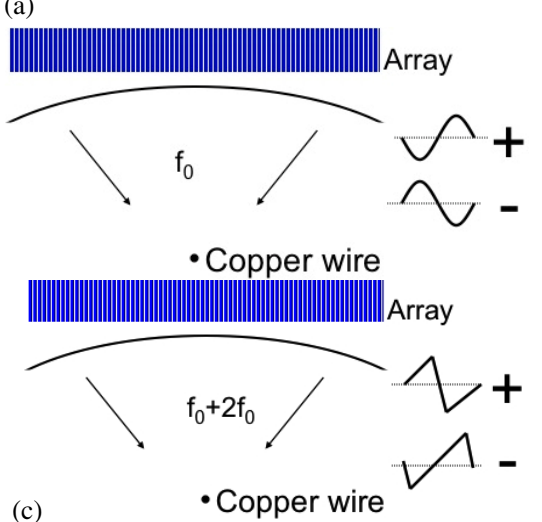

(b)

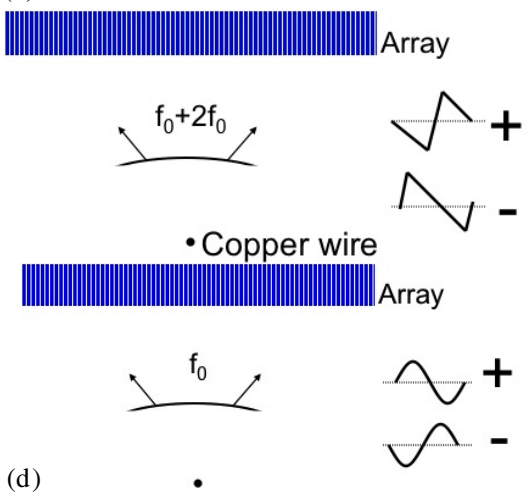

Figure 2. Methodology for adaptive cancellation of harmonics due to nonlinear propagation. (a) Calibration: straight pulses (monochromatic Gaussian) are emitted by the array through a medium. (b) Calibration: the pulses, distorted by nonlinear propagation and scattered by the copper wire, are acquired by the array. (c) Optimization: time-reversed distorted pulses are reemitted towards the copper wire. (d) Optimization: harmonic-free echoes are received by the array.

(France). In emission, the individual signals were sampled at $80 \mathrm{MHz}$ with a dynamic range of 12 bits and a maximum peak-to-peak amplitude of $100 \mathrm{~V}$. The experiments were performed using a 128 -element linear array with a $0.33 \mathrm{~mm}$ pitch and centred around $4.3 \mathrm{MHz}$ (Vermon, France).

The linearity of the electronics was tested by measuring the echo from a metal plate, placed $1 \mathrm{~cm}$ away from the array elements, which were independently emitting Gaussian pulses at various amplitudes.

During the calibration phase, the distorted pulses were acquired by collecting the ultrasound echoes from a copper wire with a diameter of $50 \mu \mathrm{m}$. The copper wire was aligned perpendicularly to the array, separated by $5 \mathrm{~cm}$ of degassed water. Delays were set to focus the array transducer on the wire. Pulses of various peak-negative pressures (0.2-2.6 MPa peak-negative pressure), frequencies (2-5 MHz) and bandwidth $(0.1-5 \mathrm{MHz})$ were emitted. Another set of pulses, opposite in phase, were also emitted for the PI experiments.

For each pulse, the echo from the wire was collected and bandpass filtered (2-7 MHz). It was then divided, in the frequency domain, by the one-way bandwidth of the transducer and the measured frequency response of the wire. This step was performed in order to isolate the acoustic effects, such as nonlinear propagation and diffraction, from effects generated by the measuring apparatus. After this deconvolution, the resulting distorted signals were time-reversed and saved for further use.

The first phase of the optimization was performed over the wire by varying the centre frequency of the pulse. The undistorted (straight) and distorted (TR-corrected signals) were emitted and the echo from the wire was collected. The received signal was analysed in the frequency domain and by summing the two pulses of PI to determine the resulting PI signal. The signal resulting in the lowest harmonics level was chosen for further experiments. Similar optimization steps were carried out with respect to the bandwidth of the pulses.

A minimization of propagation harmonics was also performed in tissue phantom at different pulse amplitudes. The phantom was prepared with $3 \%$ agar, $1.5 \%$ cellulose powder scatterers and $5 \%$ propanol (Sigma-Aldrich, USA) mixed in water. The array was placed on 


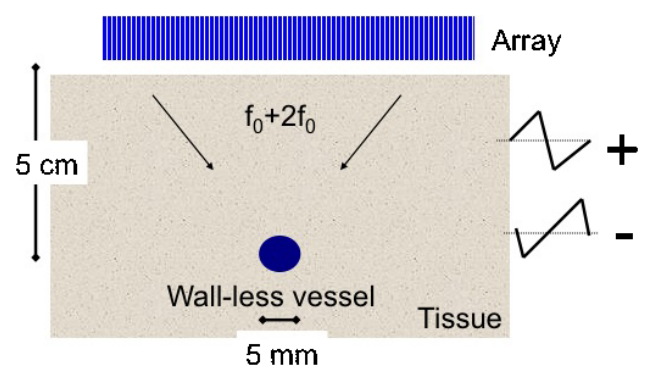

Figure 3. Setup for B-mode tissue phantom imaging using distorted pulses.

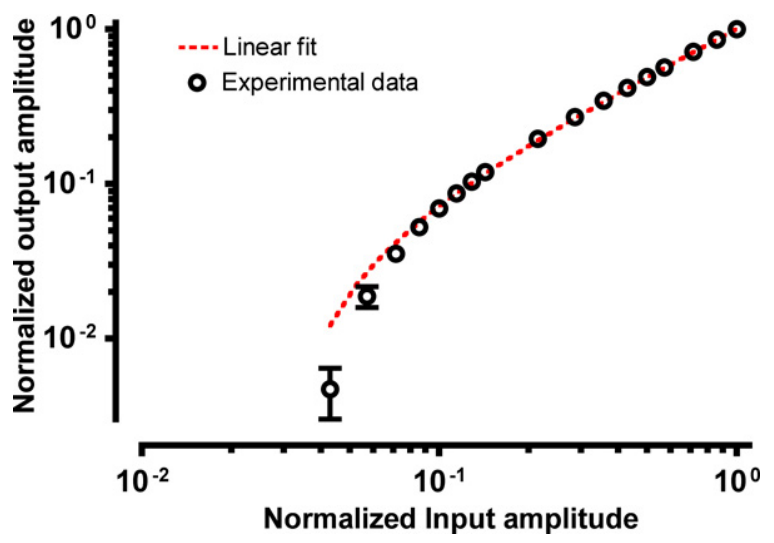

Figure 4. Linearity of the amplitude of the echo from a metal plate $1 \mathrm{~cm}$ away. Cross-transducer standard deviation is smaller than the symbols. The slope of the linear fit $\left(R^{2}=0.998\right)$ is $1.038 \pm$ 0.004 .

the phantom and was electronically focused in emission and reception at a depth of $5 \mathrm{~cm}$. Pulses with increasing levels of distortion, corresponding to echoes obtained at increasing pressures in the first phase, were emitted at various amplitudes and the resulting PI signal was minimized.

A wall-less vessel phantom was prepared by creating a tunnel, $5 \mathrm{~mm}$ in diameter, through the tissue phantom (figure 3) (Rickey et al 1995). Ultrasound contrast agents (Bracco Research, Switzerland) were diluted down to a $1 / 10000 \mathrm{v} / \mathrm{v}$ concentration in gas-equilibrated water. The solution was then flowed by gravity through the wall-less vessel phantom. Imaging was performed with the pulses optimized as per the previous step and also using straight pulses with the same pressure. These pulses were delayed following a conventional beam-forming scheme with $\mathrm{f} / 2.5$.

\section{Results}

\subsection{Emitter-receiver characterization}

Initially, the linearity of the new 128-channel 12-bit emitter receiver was examined. The amplitude of the echo from a metal plate following the emission of pulses of increasing pressure is shown in figure 4. A proportional relationship between the input and output amplitudes on individual transducers was observed between $1 \%$ and $100 \%$ power. 


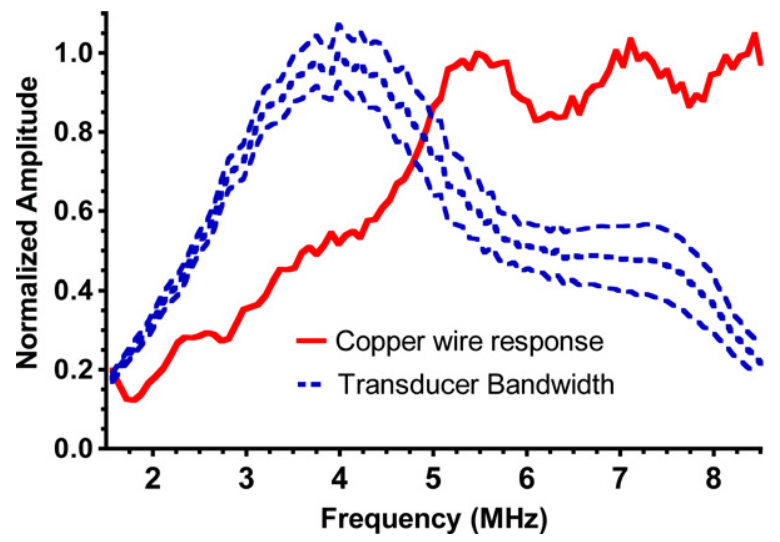

Figure 5. Average one-way bandwidth of the ultrasonic array (with inter-element standard deviation) and frequency response of the copper wire.
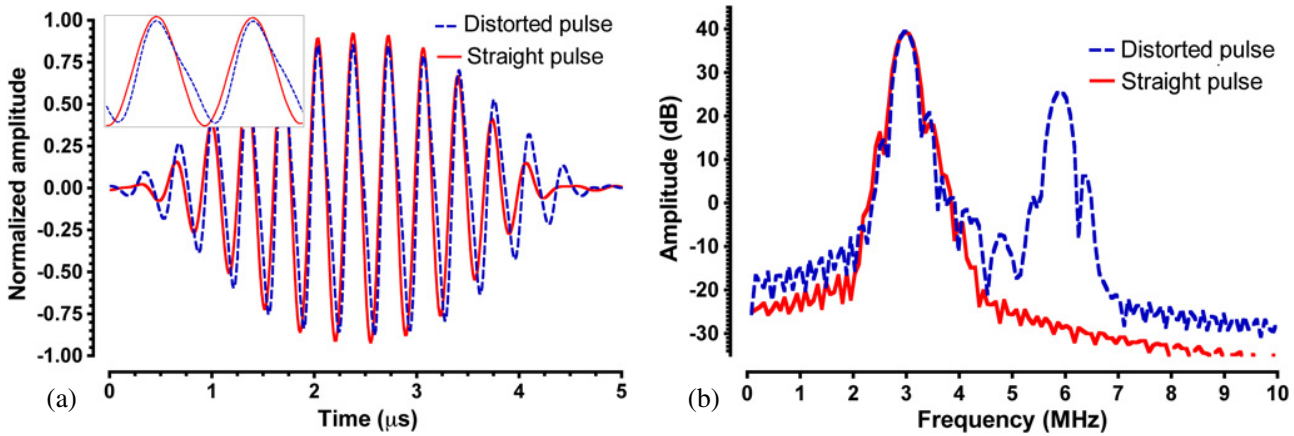

Figure 6. Example of a straight and time-reversed distorted pulse in the time (a, including a zoom over two periods) and frequency (b) domain.

Figure 5 shows the one-way bandwidth of the elements of the transducers as measured with a reflecting plate. The standard deviation between the elements reached $7 \%$ of the mean response at $4 \mathrm{MHz}$. Hence, further bandwidth correction was performed on an individual elements basis. The response of the echo from the copper wire shows a strong frequency dependence that tends to favour higher harmonics. For a $2.9 \mathrm{MHz}$ pulse, the second harmonic was scattered almost three times in excess to the fundamental.

\subsection{Calibration on a copper wire}

Pulses with increasing distortion were acquired by increasing the emitted pressure towards the wire. The straight pulse in figure 6(a) was obtained by bouncing a low pressure pulse on the wire and the distorted pulse was obtained using a high-pressure pulse. The trough of the corrected pulse was seen to lead the trough of the straight pulse. During nonlinear backpropagation, the slower moving trough was meant to catch up with the crest. This distortion was linked to the high amount of second harmonics present $(15 \mathrm{~dB}$ below the fundamental, figure 6(b)). 

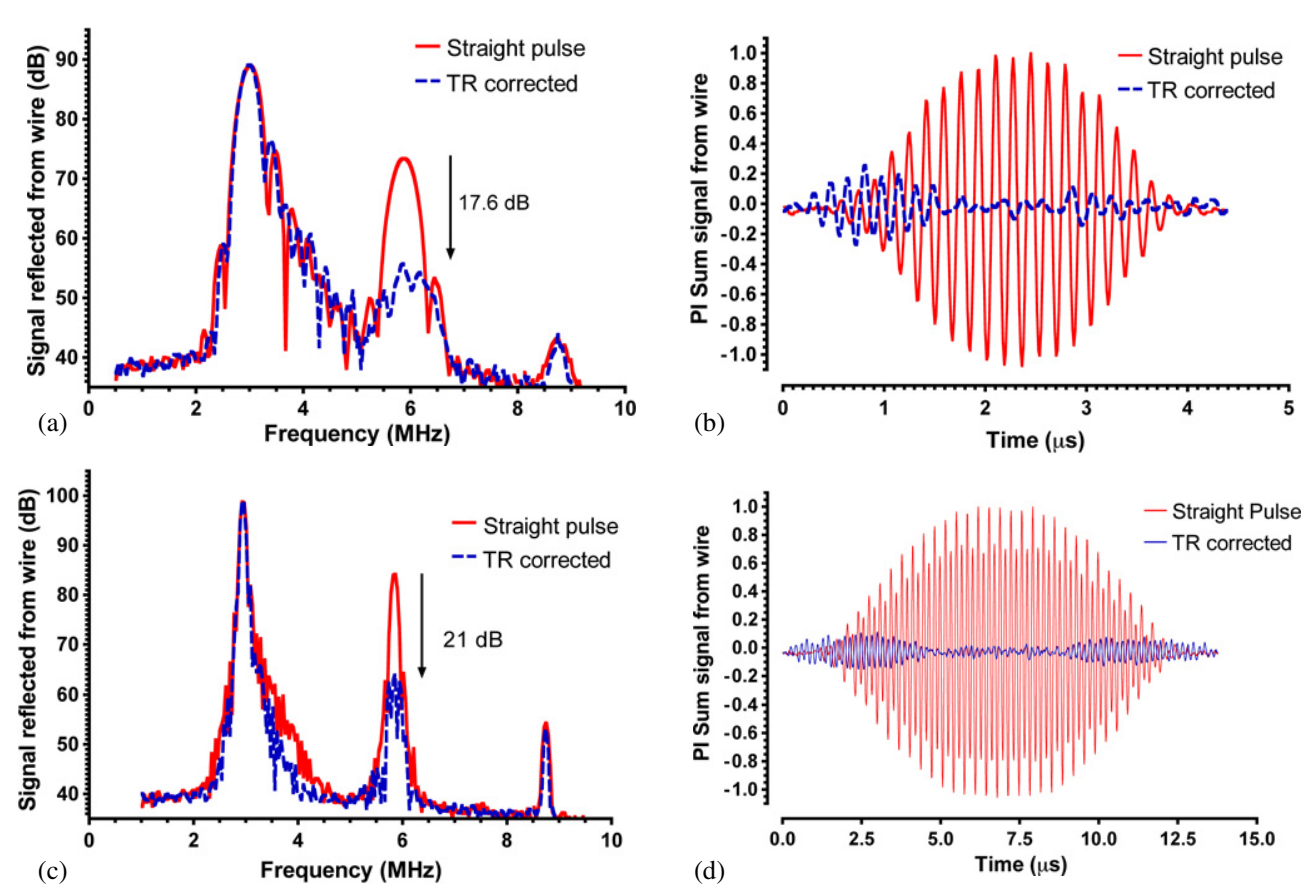

Figure 7. Reflected signal of $2.9 \mathrm{MHz}$ pulses in the frequency-domain and time-domain residuals of the pulse-inversion signal. 12-cycle (a), (b) and 50-cycle pulses (c), (d).

After the time-reversed pulses of different levels of distortion were acquired and time reversed, they were used to perform pulse-echo experiments over the copper wire. Two different ways of depicting the effectiveness of time-reversal tissue harmonics cancellation were used. First, the harmonics content of the echo from the wire was compared between the straight pulse and the TR-corrected pulses as the spectra shown in figure 7(a). In this specific case, for a pulse at $2.9 \mathrm{MHz}$ centre frequency, the correction was able to reduce the second harmonics content of the wire's echo by almost $18 \mathrm{~dB}$. Secondly, the cancellation of nonlinear propagation was also demonstrated by the residuals of the PI scheme in the time domain, which removes most of the fundamental signal. In figure 7(b), the sum of the phase-inverted pulses is compared to that of the straight pulses. The maximum amplitude of the resulting signal is reduced by a factor of 4 by the time-reversal cancellation. The correction eliminated most of the PI signal due to nonlinear propagation except for the early onset of the pulse.

Since a large fraction of the PI signal after TR correction seemed to be due to the onset of the pulse, longer pulses were also used. As shown in figure 7(c), for 50 cycle pulses, the second harmonic could be reduced by a factor of almost $22 \mathrm{~dB}$. Most of the energy in the time-corrected pulses was on the side of the pulse (figure $7(d)$ ). Isolating the central part, a nonlinear propagation cancellation of $32 \mathrm{~dB}$ was observed.

Since the PI time signal is of interest for ultrasound imaging, its root-mean-square value was calculated to assess the effectiveness of the time-reversal cancellation. This process was reiterated for 12-cycle pulses of different centre frequencies as shown in figure 8 . The most efficient pulse was centred at $2.9 \mathrm{MHz}$, with a total reduction in the time domain signal of $12 \mathrm{~dB}$. This frequency corresponded to the lower limit of the $-15 \mathrm{~dB}$ bandwidth of the transducer. The time-reversal correction was detrimental for pulses below $2.6 \mathrm{MHz}$ and above 3.5 $\mathrm{MHz}$. 


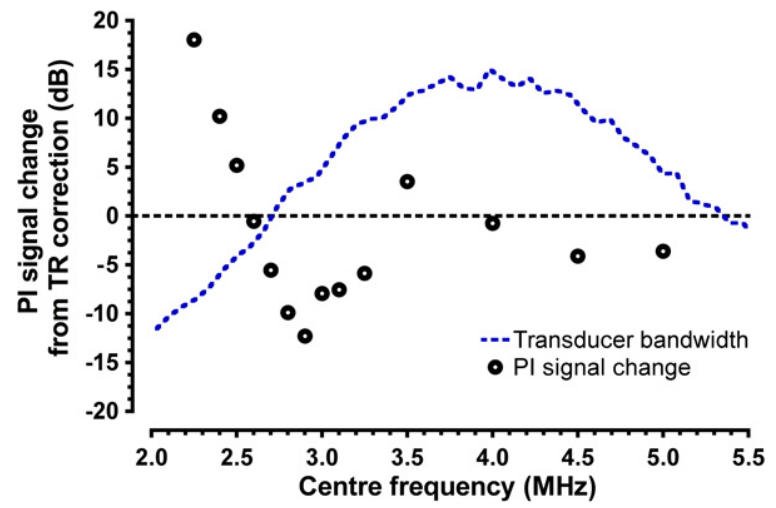

Figure 8. Frequency optimization using the reflection from a wire. The sum of the reflection of the phase-inverted pulse is compared between the straight and TR-corrected pulses.

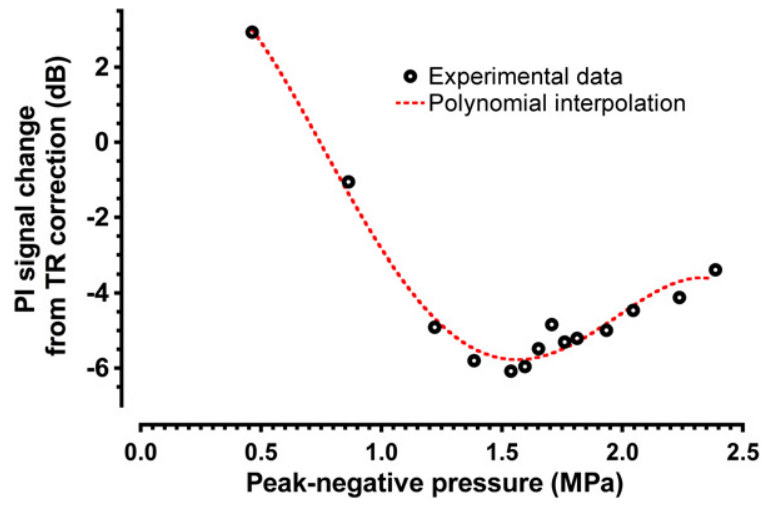

Figure 9. Optimization of the suppression of the pulse-inversion signal in the tissue phantom. The level of distortion is fixed and the input pressure is varied.

\subsection{Optimization in tissue phantom}

For a specific depth and pulse distortion level, an optimized pulse pressure is expected to minimize tissue harmonics. Figure 9 shows this minimization process for a fixed level of distortion (second harmonics/fundamental $=-13 \mathrm{~dB}$ ) and a varying peak pressure. The residual signal of the PI scheme was averaged over a window of $2 \mathrm{~cm}$ thickness at $5 \mathrm{~cm}$ depth. The effect of the distorted pulses was compared to that of the straight pulses. At lower input pressures, the time-reversal correction injected additional second harmonic signals. As the pressure was increased, the time-reversal correction became beneficial. The pressure amplitude at which the minimum was attained increased with the level of pulse distortion. Over a window of $2 \mathrm{~cm}$ in depth, the nonlinear signal was decreased by more than $6 \mathrm{~dB}$.

The PI signal is shown as a function of depth in figure 10. When straight pulses were used to image the phantom, the nonlinear PI signal increased significantly in the focal region. With respect to these pulses, the pulses corrected by time reversal showed an $8 \mathrm{~dB}$ reduction in the backscattered signal from the focal zone. 


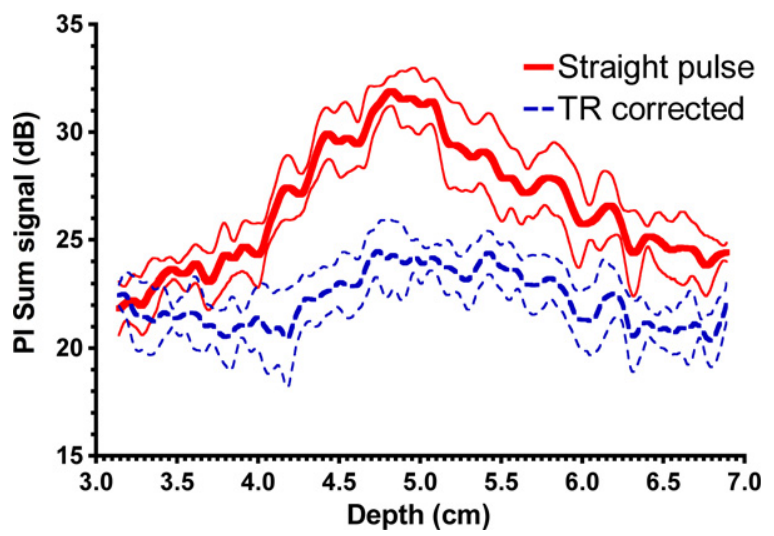

Figure 10. Depth profile of the PI signal in a tissue phantom around a single focus (located at $5 \mathrm{~cm}$ depth) with the standard deviation over several uncorrelated positions.

\subsection{Microbubble imaging}

After the minimization of the propagation harmonics, imaging of microbubbles passing through a wall-less tissue phantom was performed with the TR pulses. Figure 11(a) shows an image of the phantom taken when water was flowing in the tube with a PI sequence. The echo level was clearly reduced when the TR correction was applied (figure 11(b)). The depth profile of the backscattered signal is shown in figure 11(c). Over the imaging window, the signal reduction due to the correction reached $11 \mathrm{~dB}$.

After microbubbles were injected into the wall-less vessel phantom, the echo resulting from the PI sequence increased by $45 \mathrm{~dB}$, or $25 \mathrm{~dB}$ over the tissue phantom. The TR-corrected image showed a reduced level of tissue harmonics with no decrease in microbubble signal. The profiles in figures 11(c) and 12(c) are an average of the echo over several A-lines. The CTR increased by more than $10 \mathrm{~dB}$.

\section{Discussion}

In this study, the time-reversal process is separated in two phases. First, distorted pulses are established by observing the reflection from a copper wire in water. Then these distorted pulses are time reversed and used as imaging pulses in tissue. Another option would be to image with distorted pulses derived from theoretical models of nonlinear propagation, such as Burgers' equation (Hamilton and Blackstock 1998). The experimental derivation of the distorted pulses requires the correction of several factors. For instance, the bandwidth of the transducers restricts the spectrum of the received signal, which in turn lead to underdistorted pulses. Using narrowband pulses and carefully selecting the emission frequency such that the fundamental and the second harmonics are on the same level of the transducers' bandwidth could solve this issue. A more robust solution is to deconvolve the signal received from the wire by the one-way bandwidth of the transducers. Another problem arises from the frequency dependence of the reflection from the wire. Effectively, left uncorrected, it tends to favour higher harmonics and create overdistorted pulses.

The time-reversed distorted signals showed to be efficient at cancelling the second harmonics from the wire. Since the medium has not changed between the phase of the acquisition of the distorted pulses and the imaging phase, this is the purest form of time 


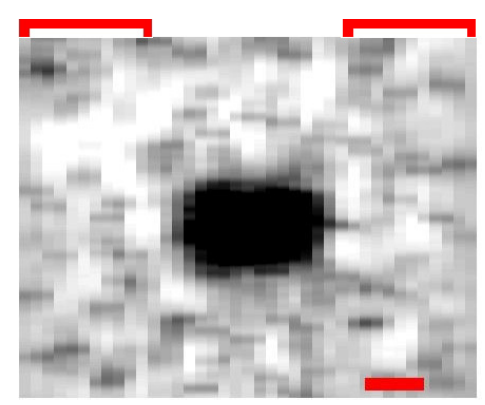

(a)

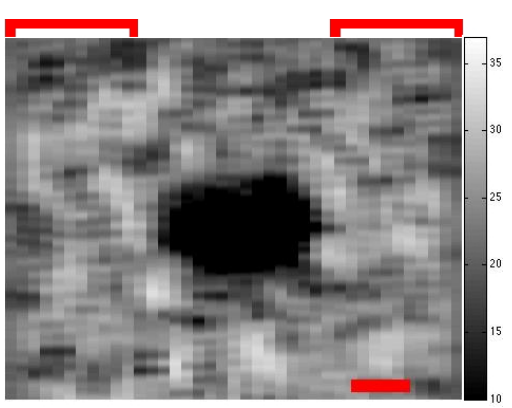

(b)

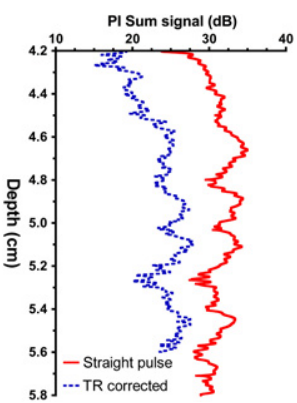

(c)

Figure 11. PI harmonic imaging of a wall-less vessel filled with water using straight pulses (a) and TR correction (b). Corresponding depth profile (c). The scale bar is $2 \mathrm{~mm}$ long.

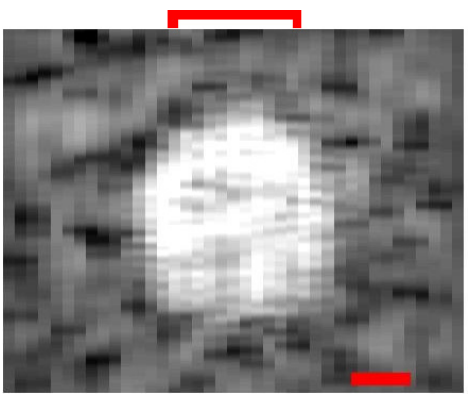

(a)

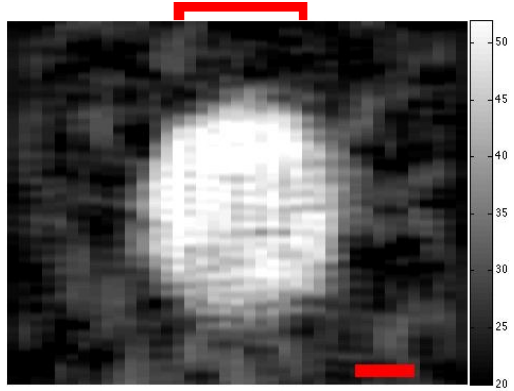

(b)

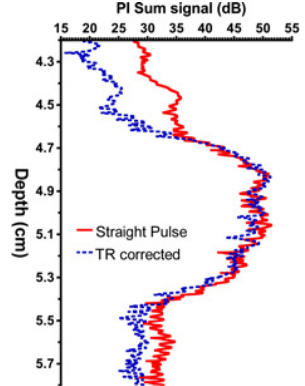

(c)

Figure 12. PI harmonic imaging of a wall-less vessel filled with a microbubble solution using straight pulses (a) and TR correction (b). Corresponding depth profile (c). The scale bar is $2 \mathrm{~mm}$ long.

reversal. For a pulse of reasonable length (12 cycles), the decrease in second harmonics can reach $17.6 \mathrm{~dB}$. However, the cancellation is incomplete, particularly at the beginning of the pulses.

This incomplete cancellation is clearer when the PI signal is observed. Indeed, when the echoes of the distorted pulses of opposite polarity are summed, only the onset and end of the pulses remains. In fact, within the central part of very long pulses, the decrease in the second harmonic level can reach $32 \mathrm{~dB}$. This could be due to the time response of the electronics driving the transducers. Consequently, longer pulses would allow better cancellation. However, to retain axial resolution, a 12-cycle pulse is preferred for imaging. Further developments in linear transmit electronics could improve the cancellation at the onset of the pulses and allow imaging with shorter pulses.

The bandwidth of the transducer is clearly important in the choice of the emitted frequencies. At the very least, the array has to be sensitive to both the fundamental and the second harmonics. Ideally, for a more complete time reversal, additional harmonics should be detectable, which is technically difficult. The optimization of the frequency shows that it is preferable for the fundamental and the second harmonic to be distributed over the two sides of the bandwidth of the transducer (figure 8). For our specific transducer, these boundaries are those of the $-15 \mathrm{~dB}$ bandwidth. 
The pressure optimization shows that there is a specific level of distortion that will cancel a wave of a specific pressure at a certain depth. This is expected since the time-reversal technique, in fact, works only on a very small region of space. Prior to the wave attaining this region, the wave is overcorrected by the distorted pulses and the TR correction adds harmonics. Following the optimal cancellation point, the wave is undercorrected and some harmonics are left. If regions with a higher nonlinearity parameter are present within the path of the ultrasound, this optimal cancellation point should move closer to the transducer.

Contrary to theoretical time reversal, where the received signal is untouched apart from the temporal flip, the experimental method involves selecting the output pressure that minimizes tissue harmonics. This is required because of the asymmetry between reception and emission electronics, but also because of absorption. Additionally, in a heterogeneous medium, cancellation is smoothed over a larger region. Such an effect is observable in the depth profile of the scattering from a tissue phantom where the PI signal is reduced over most of the focal region (figure 10). This spatial averaging reduces the absolute nonlinear cancellation, but allows the application of the technique over a longer depth of field. The way the nonlinear cancellation is measured is thus very important. If quasi-continuous waves are used on a wire in water, a very large decrease in the nonlinear propagation is observed. However, when the correction is averaged over a large depth of tissue phantom, nonlinear cancellation is more subtle, but still clearly beneficial.

The image of the wall-less vessel phantom demonstrates that the contrast between microbubbles and tissue phantom is enhanced significantly by the TR correction. The region occupied by the microbubbles is much brighter than the background tissue phantom. Clearly, the signal from the microbubbles is partly due to decorrelation between the two phase-inverted pulses coming from motion and disruption. The MI was effectively higher than the disruption threshold. However, independently of the origin of the microbubble signal, the tissue phantom signal is reduced by more than $10 \mathrm{~dB}$.

Future applications of time-reversal correction in clinical setting are limited by the sensitivity of the microbubbles. Effectively, because newer generations of microbubble scatter harmonics at lower pressures, imaging is now often performed at MI that induces only minor nonlinear propagation. However, the time-reversal technique might be of interest for the imaging of stiffer microbubbles at greater depth.

Emitting the time-reversed distorted pulses requires a wide dynamic range in emission, which is not available in current clinical scanners. Custom electronics had to be developed for such an application. Nevertheless, the apparatus' ability to emit 12-bit arbitrary waveforms on 128 independent channels can now be exploited to implement newly developed pulse sequences.

We can foresee that future ultrasound scanners will be equipped with a dial allowing the technician to control the level of distortion of the emitted pulses. This implementation would permit the cancellation of tissue harmonics coming from a specific depth in the tissue and increase the contrast from the microbubbles flowing within the deep capillaries. It would also allow the user to correct for a perceived variation in tissue nonlinearity and adapt to various tissues, from blood to adipose.

\section{Conclusion}

The goal of this study was to cancel tissue harmonics in order to improve the contrast-to-tissue ratio in microbubble imaging. The technique described is based on the time invariance of the wave equation in the nonlinear regime (Tanter et al 2001). Theoretically, this reversibility holds until the shock distance is attained and under conditions of weak absorption. This 
technique was experimentally applied on a newly developed fully programmable scanner. Harmonic cancellation of up to $18 \mathrm{~dB}$ was observed on the echo of a wire in water. In tissue, the pulse-inversion signal from tissue was reduced by up to $11 \mathrm{~dB}$, while the signal from microbubbles was maintained at the same level. Thus, the time-reversal cancellation technique could help perfusion imaging within deeper organs.

\section{Acknowledgments}

We thank Bracco Research (Switzerland) for providing the contrast agents. We also thank Vera Khokhlova for fruitful discussions.

\section{References}

Aanonsen S I and Barkve T 1984 Distortion and harmonic generation in the nearfield of a finite amplitude sound beam J. Acoust. Soc. Am. 75 749-58

Albrecht T et al 2003 Improved detection of hepatic metastases with pulse-inversion US during the liver-specific phase of SHU 508A: multicentre study Radiology 227 361-70

Bouakaz A, Frigstad S, Ten Cate F J and de Jong N 2002 Improved contrast to tissue ratio at higher harmonics Ultrasonics 40 575-8

Burns P N and Wilson S R 2006 Microbubble contrast for radiological imaging: 1. Principles Ultrasound Q. 22 5-13

Cobbold R S C 2007 Foundations of Biomedical Ultrasound (Oxford: Oxford University Press)

Cosgrove D 2003 Angiogenesis imaging-ultrasound Br. J. Radiol. 76 S43-S49

de Jong N, Emmer M, Chin C T, Bouakaz A, Mastik F, Lohse D and Versluis M 2007 Compression-only behavior of phospholipid-coated contrast bubbles Ultrasound Med. Biol. 33 653-6

de Jong N, Frinking P J A, Bouakaz A and Ten Cate F J 2000 Detection procedures of ultrasound contrast agents Ultrasonics 38 87-92

Hamilton M F and Blackstock D T 1998 Nonlinear Acoustics (New York: Academic)

Krishnan K B and Thomenius K E 2008 Improved contrast ultrasound with tissue harmonic minimizing pulse IEEE Trans. Ultrason. Ferroelectr. Freq. Control 55 249-53

Krishnan S, Hamilton J D and O'Donnell M 1998 Suppression of propagating harmonic in ultrasound contrast imaging IEEE Tans. Ultrason. Ferroelectr. Freq. Control 45 704-11

Lencioni R, Cioni D and Bartolozzi C 2002 Tissue harmonic and contrast-specific imaging: back to gray scale in ultrasound Eur. Radiol. 12 151-65

Miller A P and Nanda N C 2004 Contrast echocardiography: new agents Ultrasound Med. Biol. 30 425-34

Pasovic M, Basset O, van der Steen A F W, Cachard C and de Jong N 2008 Local reduction of second harmonic due to ultrasound propagation: simulation and feasibility 13th European Symp. on Ultrasound Contrast Imaging (Rotterdam, the Netherlands)

Phillips P and Gardner E 2004 Contrast-agent detection and quantification Eur. Radiol. Suppl. 14 4-10

Rickey D W, Picot P A, Christopher D A and Fenster A 1995 A wall-less vessel phantom for Doppler ultrasound studies Ultrasound Med. Biol. 21 1163-76

Simpson D H, Burns P N and Averkiou M A 2001 Techniques for perfusion imaging with microbubble contrast agents IEEE Trans. Ultrason. Ferroelectr. Freq. Control 48 1483-94

Simpson D H, Chin C T and Burns P N 1999 Pulse inversion Doppler: a new method for detecting nonlinear echoes from microbubble contrast agents IEEE Trans. Ultrason. Ferroelectr. Freq. Control $46372-82$

Tanter M, Thomas J L, Coulouvrat F and Fink M 2001 Breaking the time reversal invariance in nonlinear acoustics Phys. Rev. E 64 1-7

Tranquart F, Grenier N, Eder V and Pourcelot L 1999 Clinical use of ultrasound tissue harmonic imaging Ultrasound Med. Biol. 6 889-94

Wei K, Jayaweera A R, Firoozan S, Linka A, Skyba D M and Kaul S 1998 Quantification of myocardial blood flow with ultrasound-induced destruction of microbubbles administered as a constant venous infusion Circulation 97 473-83 